\title{
Author Correction: Arylmethylamino steroids as antiparasitic agents
}

Reimar Krieg ${ }^{1}$, Esther Jortzik² ${ }^{2}$ Alice-Anne Goetz ${ }^{3}$, Stéphanie Blandin³, Sergio Wittlin ${ }^{4,5}$, Mourad Elhabiri6 Mahsa Rahbari ${ }^{2}$, Selbi Nuryyeva ${ }^{6,7}$, Kerstin Voigt ${ }^{8}$, Hans-Martin Dahse $^{8}$, Axel Brakhage ${ }^{8}$, Svenja Beckmann ${ }^{9}$, Thomas Quack $^{9}$, Christoph G. Grevelding ${ }^{9}$, Anthony B. Pinkerton ${ }^{10,11}$, Bruno Schönecker ${ }^{12}$, Jeremy Burrows ${ }^{13}$, Elisabeth Davioud-Charvet ${ }^{6}$, Stefan Rahlfs ${ }^{2} \&$ Katja Becker ${ }^{2}$

Correction to: Nature Communications https://doi.org/10.1038/ncomms14478, published online 17 February 2019.

This Article contains errors in Figure 1a. The chemical structure of compound $\mathbf{9}$ is incorrect. The configurational assignments for compounds 3, 5, 7 and 10 are incorrectly labeled as '17alpha' and should be labelled as '16alpha'. The configurational assignments for compounds 4, 6, 8 and $\mathbf{1 1}$ are incorrectly labeled as '17beta' and should be labelled as '16beta'. The configurational assignments for compounds $\mathbf{1 3}$ and $\mathbf{1 4}$ are incorrectly labeled as '17alpha' and '17beta' and should be labelled as '3beta' and '3alpha', respectively. The correct version of Fig. 1a is:
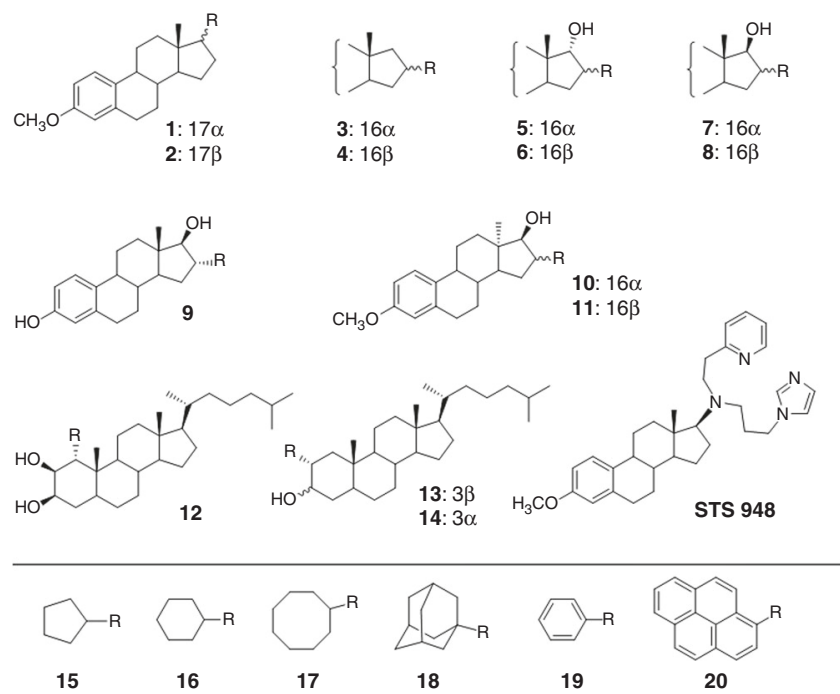

Fig. 1

\footnotetext{
${ }^{1}$ Institute of Anatomy II, University Hospital Jena, Teichgraben 7, 07743 Jena, Germany. ${ }^{2}$ Biochemistry and Molecular Biology, Interdisciplinary Research Centre, Justus Liebig University Giessen, Heinrich Buff Ring 26-32, 35392 Giessen, Germany. ${ }^{3}$ Université de Strasbourg, CNRS, Inserm, MIR UPR9022/ U963, F-67000 Strasbourg, France. ${ }^{4}$ Swiss Tropical and Public Health Institute, Socinstrasse 57, PO Box, 4002 Basel, Switzerland. ${ }^{5}$ University of Basel, Petersplatz 1, 4001 Basel, Switzerland. ${ }^{6}$ UMR 7509 Centre National de la Recherche Scientifique and University of Strasbourg, European School of Chemistry, Polymers and Materials (ECPM), 25, rue Becquerel, F-67087 Strasbourg, France. ${ }^{7}$ New York University Abu Dhabi, PO Box, 129188 Abu Dhabi, UAE. 8 Leibniz Institute for Natural Product Research and Infection Biology-Hans Knöll Institute (HKI), Adolf-Reichwein-Strasse 23, 07745 Jena, Germany. ${ }^{9}$ Institute of Parasitology, Biomedical Research Centre, Justus Liebig University Giessen, Schubertstrasse 81, 35392 Giessen, Germany. ${ }^{10}$ Conrad Prebys Center for Chemical Genomics, Sanford-Burnham-Prebys Medical Discovery Institute, 10901 North Torrey Pines Road, La Jolla, California 92037, USA. ${ }^{11}$ Conrad Prebys Center for Chemical Genomics, Sanford-Burnham-Prebys Medical Discovery Institute, 6400 Sanger Road, Orlando, Florida 32827, USA.

12 Institute of Organic and Macromolecular Chemistry, Friedrich Schiller University Jena, Humboldtstrasse 10, 07743 Jena, Germany. ${ }^{13}$ Medicines for Malaria Venture, 20 Route de Pré-Bois, 1215 Geneva 15, Switzerland. Correspondence and requests for materials should be addressed to K.B. (email: katja. becker@uni-giessen.de)
} 
The error has not been corrected in the PDF or HTML versions of the Article.

Published online: 08 July 2019

(c) (i) Open Access This article is licensed under a Creative Commons Attribution 4.0 International License, which permits use, sharing, adaptation, distribution and reproduction in any medium or format, as long as you give appropriate credit to the original author(s) and the source, provide a link to the Creative Commons license, and indicate if changes were made. The images or other third party material in this article are included in the article's Creative Commons license, unless indicated otherwise in a credit line to the material. If material is not included in the article's Creative Commons license and your intended use is not permitted by statutory regulation or exceeds the permitted use, you will need to obtain permission directly from the copyright holder. To view a copy of this license, visit http://creativecommons.org/licenses/by/4.0/.

(c) The Author(s) 2019 